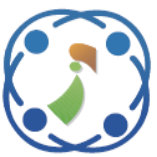

\title{
Multilevel thresholding and Morphological Relationship Approach for Automatic Detection of Anterior and Posterior Commissure in Mid-sagittal Brain MRI
}

\author{
Khairiyyah Nur Aisyah ${ }^{1}$ \\ Chastine Fatichah ${ }^{1 *}$ \\ Riyanarto Sarno ${ }^{1}$ \\ ${ }^{1}$ Department of Informatics, Institut Teknologi Sepuluh Nopember, Indonesia \\ * Corresponding author's Email: chastine@if.its.ac.id
}

\begin{abstract}
Most of neuroimaging applications tend to still rely on expert knowledge in determining anatomies of the brain. For example in Parkinson's disease surgery, detection of the anterior commissure (AC) and posterior commissure (PC) are still done manually by doctors. Previously, various methods have been developed related to the automatic detection of AC and PC. However, the majority of previously methods have several drawbacks, such as only compatible on $\mathrm{T} 1-\mathrm{W}$ or $\mathrm{T} 2-\mathrm{W}$, only compatible for data with the same matrix size, and requires a time-consuming training process. This study proposes a new strategy by combining a multilevel thresholding and morphological relationships approach for automatic detection of $\mathrm{AC}$ and $\mathrm{PC}$. The process divided into 4 main stages: preprocessing, multilevel thresholding, segmentation, and detection of AC and PC. The segmentation is performed on several anatomies of the brain including corpus callosum, fornix, and colliculus. From the experiment, it can be concluded that the use of multilevel thresholding and morphological relationship was successfully detecting AC and PC with the mean error were $1.02 \mathrm{~mm}$ and $1.06 \mathrm{~mm}$, respectively. The proposed method can perform an automatic detection of $\mathrm{AC}$ and $\mathrm{PC}$ with simply algorithm, does not require a large of diverse data sets for the training process, without training process that takes up time, and reliable on the diversity of MRI since it is compatible for T1-W and T2-W with various matrix sizes of $256 \times 256$ and $512 \times 512$ pixels which cannot be handled by previous researches.
\end{abstract}

Keywords: Anterior commissure, Brain MRI, Colliculus, Corpus callosum, Fornix, Otsu multilevel thresholding, Parkinson, Posterior commissure.

\section{Introduction}

Human brain is an organ with the same anatomical structure but has different variations in shape, size, and position of each individual brain structure. One of the anatomies of the brain that has an anatomical structure which difficult to observe in detail with conventional MRI is anterior commissure (AC) and posterior commissure (PC) [1]. AC and PC are a collection of transverse fibres connecting the two hemispheres of the brain and helps the exchange of information between the two [2]. Some roles of AC and PC include mapping of the human brain [3], medical image processing [4], and assisting in stereotactic neurosurgery $[5,6]$.

In some stereotactic neurosurgery, such as in Parkinson's disease surgery, detection of the anatomies is still done manually by doctor. The majority of existing neuroimaging applications tend to rely on expert knowledge in determining the anatomy of the brain, for example in determining the location of AC and PC which are vital organs in the process of surgery. In stereotactic neurosurgical procedures, target detection is based on a standard coordinate system introduced by Talairach and Tournoux. This coordinate system is referenced to $\mathrm{AC}$ and $\mathrm{PC}$ [7]. Mid-commissural point (MCP), or the midpoint of a line connecting $\mathrm{AC}$ and $\mathrm{PC}$, is the most commonly used centre for stereotactic targeting. Thus, the variability in manual selection of $\mathrm{AC}$ and PC greatly impact on the detection of targets in each brain region. Manual detection of some vital anatomy of the brain certainly requires expertise, takes a lot of time, and potentially diversity of detection between experts [8]. Therefore, the development of an automatic detection of anatomies that plays an 
important role in stereotactic neurosurgery are needs to be developed.

Previously, various methods have been developed related to the automatic detection of AC and PC. Zhang et al. [9] conducted an automatic detection of $\mathrm{AC}$ and $\mathrm{PC}$ using anatomic and radiological approaches. The detection process performed by identifying the relationship between $\mathrm{AC}$ and $\mathrm{PC}$ with the corpus callosum (CC), fornix (Fo), brainstem (BS), third ventricle (V3), and aqueduct (Aq) in the mid-sagittal plane of the brain. Although it is mentioned that the proposed method is quite fast and accurate, it cannot handle the diversity of MRI since it only compatible on T2-W MRI with the matrix sizes of $320 \times 248$ pixels. Another research related to automatic detection of AC and PC was carried out by Ardekani and Bachman [10]. The algorithm proposed uses a model-based approach so that it does not require segmentation of corpus callosum, not depend on contrast diversity, and compatible with T1-W or T2-W MRI. However, based on the test results on $256 \times 256 \mathrm{MRI}$, the mean error of $\mathrm{T} 2-\mathrm{W}$ is higher because the training process is only used T1-W MRI data. It can be concluded that the proposed method is highly depends on training data. Thus in order to get the maximum training model, a large enough data sets with diverse variations are needed. In addition, the training process also tends to be complex with the amount of information that needs to be collected from each training data. Liu et al. [11] proposed an automatic localization of the anterior commissure, posterior commissure, and mid-sagittal plane in MRI using regression forests. The experiment was applied in 100 T1-W MRI data with a matrix sizes of $256 \times 256$ pixel. Although the proposed method is capable of detecting $\mathrm{AC}$ and $\mathrm{PC}$ with low error values, the paper states that all of the data sets are MRI data with similar poses, low orientation differences, and low position differences. In addition, the training process that requires diversity of MRI is also needed. The last, [12] conducted an automatic detection of AC dan PC using multi-task deep neural networks. It combined a point-detection and an angle-detection model to localizes AC and PC and determines the angulation of the line connecting these points. Since it used a neural network, a large enough dataset is needed for the training process.

The small size of AC and PC, varying pixel intensity, also the similar anatomical structure of anatomies around $\mathrm{AC}$ and PC make detection of both landmarks are quite difficult. This causes the majority of previous methods still have drawbacks in handling the diversity of MRI data, such as compatible for only a type of data (T1-W or T2-W), only compatible to data with the same matrix sizes, and requires training processes that result in a dependency on the training data used.

This study proposes a new strategy that combines multilevel thresholding and morphological relationship approach to perform an automatic detection of anterior commissure (AC) and posterior commissure (PC) on mid-sagittal brain MRI. The process in this study divided into four main stages: preprocessing, cluster selection using Otsu multilevel thresholding, segmentation, and detection of $\mathrm{AC}$ and PC. Morphological characteristics such as area, height, width, centroid, and the morphological relationship between one anatomy to others are first performed an experiment that will be used as a rule in segmentation. The segmentation is carried out on several parts of the brain: corpus callosum, fornix, and colliculus. The major contributions of this paper are as follows:

1) Proposed a new strategy for automatic segmentation of corpus callosum, fornix, and colliculus using a morphological relationship approach.

2) Proposed a new strategy that perform an automatic detection of AC and PC with simply algorithm, does not require a lot of diverse data sets for the training process, compatible to a variety of modalities such as T1-W and T2-W, and reliable to data with the various matrix sizes that cannot handled by the previous researches.

This paper is organized as follows. Section 2 discussed the basic concepts underlying this research. The proposed method is presented in Section 3. The experiment and results of the automatic detection of $\mathrm{AC}$ and $\mathrm{PC}$ are discussed in Section 4. The last, the conclusion of this research is presented in Section 5 .

\section{Literature review}

\subsection{Anterior commissure (AC) and posterior commissure (PC)}

Magnetic Resonance Imaging (MRI) is a medical imaging technique that is widely used to explore detailed information related to anatomy and the function of various organs in the body, both in terms of health and disease [13]. The results of brain MRI scans have three dimensions including axial, sagittal, and coronal, where mid-sagittal is the slice that will be used in this study to detect the location of $A C$ and PC. Anterior commissure (AC) itself is a relatively small fibre track with a diameter between $3.5-4.3$ $\mathrm{mm}$ that is visible on structural MRI in the midsagittal plane. In comparison to the corpus callosum, 
$\mathrm{AC}$ is much smaller in size, which is only about $1 \%$ of the size of the corpus callosum [14]. AC can generally be found at the tip of the sagittal image of the fornix in the mid-sagittal plane (MSP) [10], while posterior commissure (PC) is located in the inferior pineal lamina and it is one of the commissural fibres of the brain that has an important role in pupillary light reflex [15].

\subsection{Corpus callosum, fornix, and colliculus}

Based on the anatomical location of AC and PC, segmentation of several parts of the brain related to $\mathrm{AC}$ and PC is carried out to facilitate the process of detection, include corpus callosum, fornix, and colliculus. Corpus callosum is located in the centre of the human brain that connects the two hemispheres of the brain and has an important role in information exchange between the left and right cerebral hemispheres [16]. The mid-sagittal of the MRI completely portray the anatomical structure of the corpus callosum [17]. Fornix is the major outflow tract of white matter [18]. Fornix forms the letter ' $\mathrm{C}$ ' which projects the posterior hippocampus to the septum and hypothalamus [19] which attached to the inside surface of the corpus callosum (CC). The pathology that involving the fornix will be affected to the memory. Colliculus is part of the brain that divided into two parts, namely superior colliculus and inferior colliculus. Superior colliculus deals with behaviour such as target selection, attention, and decision making [20], while inferior colliculus is the principal midbrain nucleus of the auditory pathway. It is the main target for non-auditory input to the auditory system [21]. The location of AC, PC, corpus callosum, fornix, and colliculus on mid-sagittal MRI are shown in Fig. 1.

\subsection{Otsu multilevel thresholding}

Thresholding is a method that is widely used in image segmentation, both in the medical [22, 23] and non-medical [24] subjects. In computer vision and image processing, the Otsu method is commonly used for image thresholding or simply determines the threshold intensity value to split the image into two classes, background, and foreground. The determination of the threshold is done by minimizing intra-class (variance within class) and maximizing inter-class (variance between classes). In its development, the Otsu method has been developed into Otsu multilevel thresholding [25]. Supposed that pixels of image are divided into 2 classes, $C_{0}$ and $C_{1}$ by threshold at level $k, C_{0}$ denotes pixels with levels $[1, \ldots, k]$, and $C_{1}$ denotes pixels with levels $[k+$

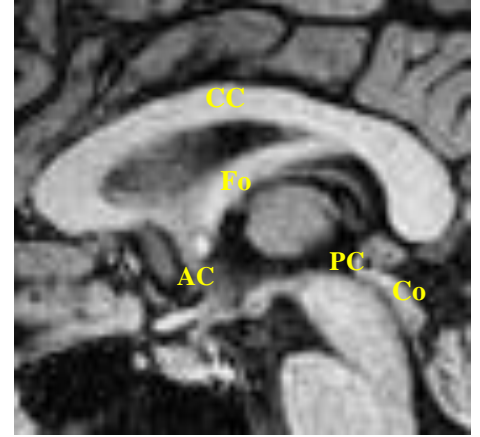

Figure. 1 Corpus callosum (CC), fornix (Fo) colliculus (Co), anterior commissure (AC), and posterior commissure (PC) on mid-sagittal MRI

$1, \ldots, L]$. Then to evaluate the "goodness" of the threshold at level $k$, the discriminant measure $(\eta)$ obtained by using Eq. (1) with $\sigma_{B}^{2}$ is inter-class variance and $\sigma_{T}^{2}$ is the total variance of levels [25].

$$
\eta=\frac{\sigma_{B}^{2}}{\sigma_{T}^{2}}
$$

The optimal threshold $k^{*}$ obtained with calculating the maximum $\eta$, or equivalently maximize $\sigma_{B}^{2}$. The value of $\sigma_{B}^{2}$ for each $k$ level can be calculated using Eq. (2). $\omega(k)$ is the probability of the occurrence in class $k, \mu(k)$ is the cumulative moment on histogram up to $k$ level, and $\mu_{T}$ is the total mean level of image [25].

$$
\sigma_{B}^{2}(k)=\frac{\left[\mu_{T} \omega(k)-\mu(k)\right]^{2}}{\omega(k)[1-\omega(k)]}
$$

\section{Proposed method}

First, Otsu multilevel thresholding is applied to mid-sagittal MRI in order to group objects into clusters. Cluster selection is then performed to reduce objects in mid-sagittal MRI to facilitate the segmentation process on the next stage. The major structures surrounding AC and PC include corpus callosum, fornix, and colliculus are firstly segmented to locate the positions of the commissures. Morphological characteristics such as area, height, width, centroid, and the morphological relationship of corpus callosum and colliculus are first performed an experiment which will be used as the rules in the segmentation process. Next, each detected object on selected clusters will be matched by the rules. Object that best matches the rules will be segmented as corpus callosum, fornix, or colliculus. After those 3 anatomies have been segmented, the last step is the localization of AC and PC. Localization of AC is done by detecting the meeting point of corpus callosum and fornix, while localization of $\mathrm{PC}$ is done by detecting the position of the top of the superior 
colliculus. Generally speaking, the proposed method is divided into 4 main stages: preprocessing, multilevel thresholding, segmentation, and detection of $\mathrm{AC}$ and $\mathrm{PC}$ as describes in Fig. 2.

\subsection{Preprocessing}

In this study, the preprocessing stage was divided into 2: contrast enhancement and cropping. Original MRI data tends to have low contrast, thus in order to clarify the image and simplify the process of segmentation, increasing the contrast needs to be done. A comparison of MRI data before and after the contrast enhancement is shown in Fig. 3.

The next step of preprocessing is cropping. The cropping process is done by cutting $\frac{1}{5}$ part of the image at the top, bottom, right side, and left side. The entire process of cropping is done automatically by the system. This step aims to remove the skull that surrounds the brain and appears on mid-sagittal MRI. This cropping process can also regarded as a process

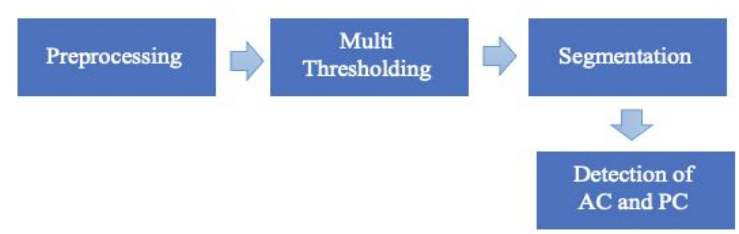

Figure. 2 Flowchart of the proposed method

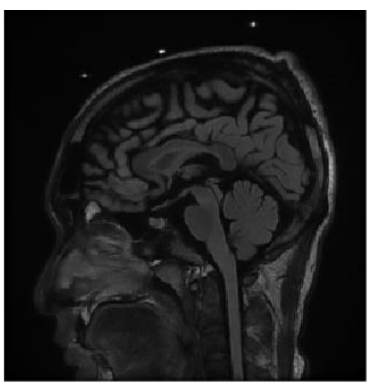

(a)

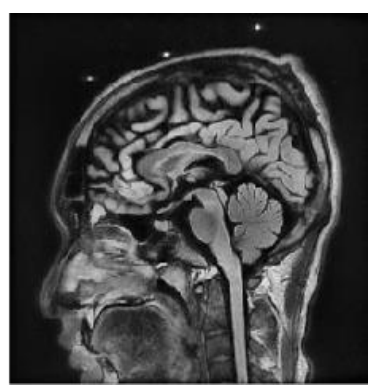

(b)
Figure. 3 Preprocessing: (a) original mid-sagittal MRI and (b) mid-sagittal MRI after contrast enhancement

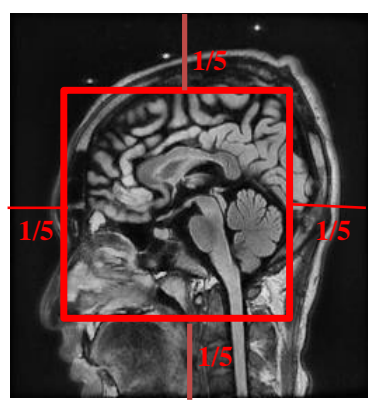

(a)

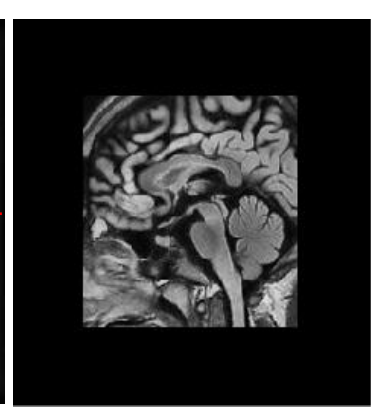

(b)
Figure. 4 Cropping: (a) mid-sagittal MRI before cropping and (b) mid-sagittal MRI after cropping of skull stripping. The result of the cropping process is shown in Fig. 4.

\subsection{Multilevel thresholding}

The thresholding method used in this study is an Otsu multilevel thresholding with 3 threshold values. Cluster selection is then performed by selecting 2 clusters, in this study called segment 1 and segment 2.

Segment 1 is the red region of a multilevel thresholding result that pixel values are greater than the third threshold value. While segment 2 is the yellow region that has pixel values between the second threshold value and the third threshold value. The result of the cluster selection is shown in Fig. 5.

In this case, dividing clusters into segment 1 and segment 2 is only applied on T2 MRI or image with $256 \times 256$ matrix sizes, while in T1 MRI or image with 512 x 512 matrix sizes, segmentation is done without the need of cluster selection.

In segment 1 and segment 2, object detection is done by using the connected component method with 8-connected neighbourhood. Set properties of each detected object will be calculated including area, centroid, and bounding box. The bounding box itself is divided into 4: left, top, width, and height. Set properties on each object will be used as the basis in creating rules. The rules will be applied to corpus callosum, fornix, and colliculus segmentation process.

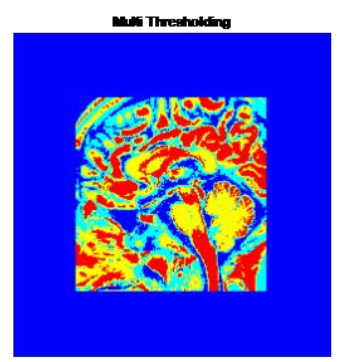

(a)

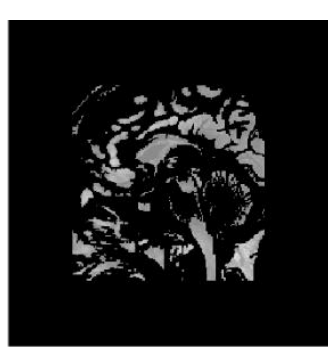

(b)

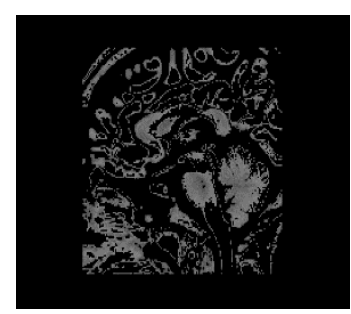

(c)

Figure. 5 Multilevel thresholding process: (a) the result of multilevel thresholding, (b) segment 1 , and (c) segment 2 
Table 1. Pseudocode for the segmentation of corpus callosum and fornix

\begin{tabular}{c}
\hline $\begin{array}{l}\text { Algorithm 1: Rules for corpus callosum and fornix } \\
\text { segmentation }\end{array}$ \\
\hline input : obj: Detected objects in segment 1, size img $:$ \\
image dimension (x-axis), centroid $(x):$ \\
centroid of object (x-axis), centroid $(y):$ \\
centroid of object (y-axis), width: the width \\
of object, height: the height of object.
\end{tabular}

output : object detected as corpus callosum and fornix

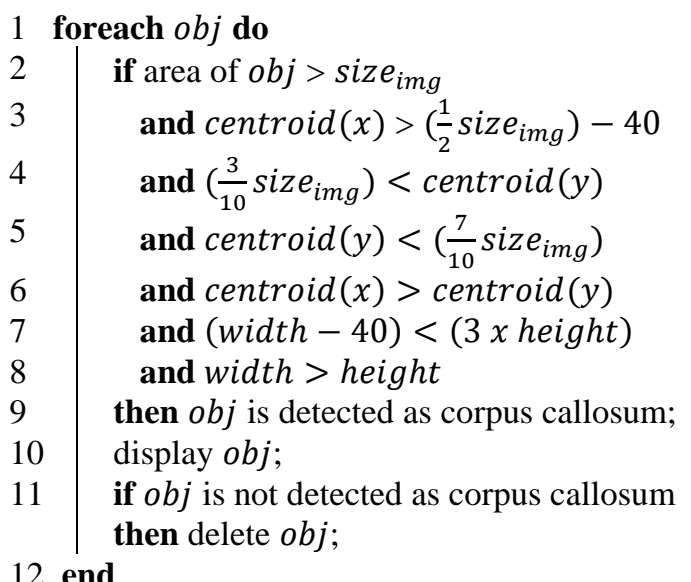

\subsection{Segmentation}

Previously, we have conducted a manual experiment on several data sets to find the morphological characteristics of corpus callosum, fornix, and colliculus, which are then used as a rule on the segmentation process that provides in Table 1 and Table 2. First, the segmentation process is performed on the corpus callosum and fornix as a single object. At this step, the segmentation process is performed on the segment 1 . The pseudocode and the rules used to segment the corpus callosum and fornix based on the morphological relationship are shown in Table 1. If there are several objects that identified as corpus callosum - fornix, then the object with the smallest value of $\mathrm{x}$-centroid will be considered as the object. The next step is establish a ROI (Region of Interest) for the segmented corpus callosum - fornix. ROI is formed by detecting automatically the right, left, top, and bottom coordinates of the segmented object and increasing 10 pixels on each side. An example of the segmented corpus callosum - fornix in segment 1 and its ROI are shown in Fig. 6. The segmentation process is then continued in segment 2 to complete the imperfect shape of the corpus callosum - fornix. ROI in segment 2 formed by copying the ROI coordinates in segment 1 , thus each segment 1 and segment 2 has ROI with the same location and size. All the objects within ROI in segment 2 will be displayed, while the entire

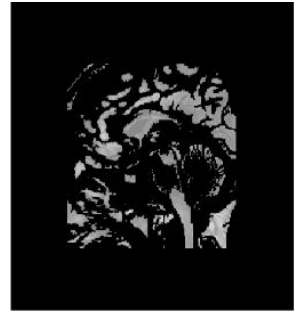

(a) (b) (c)

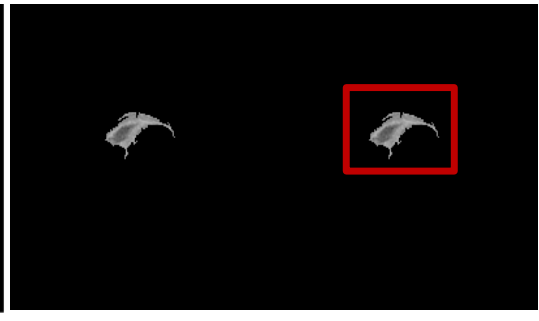

Figure. 6 Segmentation of corpus callosum - fornix: (a) segment 1, (b) segmented corpus callosum - fornix, and (c) ROI of the corpus callosum - fornix

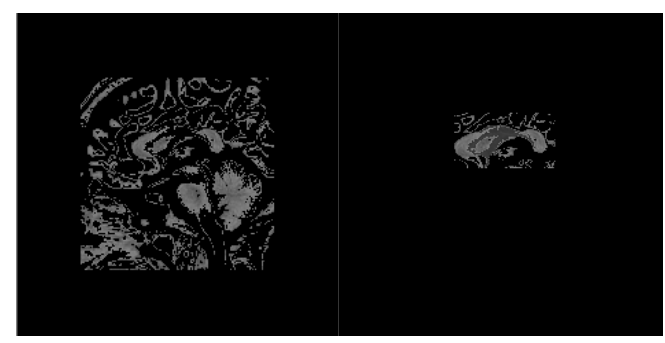

(a)

(b)

Figure. 7 Segmentation of corpus callosum - fornix: (a) segment 2 and (b) unification of objects within ROI of segment 1 and segment 2

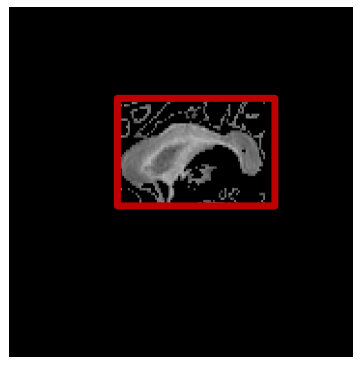

(a)

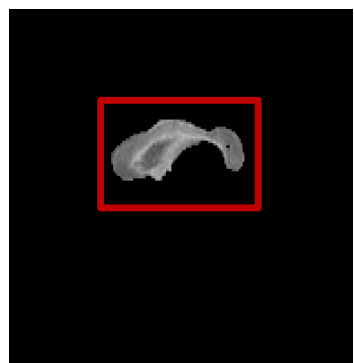

(b)
Figure. 8 :(a) Corpus callosum - fornix with noise and

(b) perfect shape of the corpus callosum - fornix

objects outside the ROI will automatically deleted. The remaining objects in segment 2 are then combined with the objects within ROI in segment 1 to form a perfect shape of corpus callosum - fornix. The result of this step is shown in Fig. 7.

The next step is to eliminate small objects around the corpus callosum - fornix. A calculation of area of each object is calculated. Object with the largest area will be considered as the corpus callosum - fornix, while all another small objects will automatically deleted. The removal of small objects attached to the corpus callosum - fornix is also performed to perfect the shape of the corpus callosum - fornix. The result of this step is shown in Fig. 8.

The segmentation process is continued by detecting colliculus. For each object in segment 1, a rule-based on morphological characteristics as shown in Table 2 will be applied. Objects that comply with the rules will be detected as colliculus, while objects 
Table 2. Pseudocode for the segmentation of colliculus
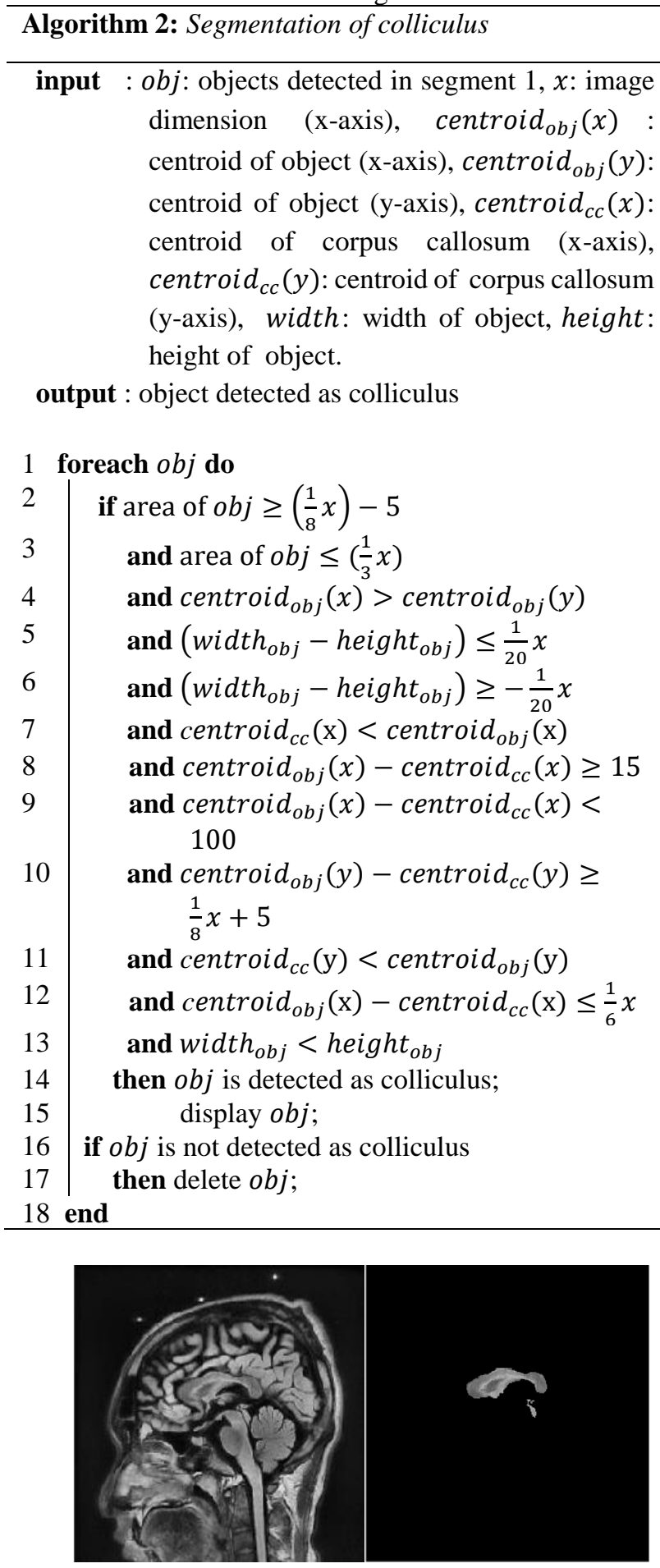

(a)

(b)

Figure. 9 Results of segmentation: (a) mid-sagittal MRI data and (b) results of automatic segmentation of corpus callosum, fornix, and colliculus

that are not eligible will automatically be removed. If none of object is detected as colliculus, it is possible that colliculus on mid-sagittal MRI is connected by the brainstem. Therefore, it is necessary to perform the optional step in order to separate pixels that connect between them. Re-segmentation is then performed using the morphological relationship that has been determined. If more than one colliculus is detected, then object with the smallest value of $\mathrm{x}$ centroid will be considered as a colliculus.

\subsection{Automatic detection of AC and PC}

The last step of this research is the automatic detection of AC and PC. Basically, AC is located at the meeting point between the corpus callosum and fornix, while the colliculus is located anteriorly to the superior colliculus. In more detail, the pseudocode for automatic detection of $\mathrm{AC}$ and $\mathrm{PC}$ in this research are shown in Table 3 and Table 4.

The next step is to re-correct the position of AC and PC. First, the coordinate of detected AC from the previous stage will be considered as point $(x, y)$. The search region is formed from point $(x, y-1)$ until point $(x+3, y+2)$. The last pixel of the search region which value is greater than the third threshold will be detected as the new coordinate of AC. For re-correct the position of PC, the coordinate of the detected PC from the previous stage is considered as point $(x, y)$. Search region then

Table 3. Pseudocode for automatic detection of AC Algorithm 3: Automatic detection of $A C$

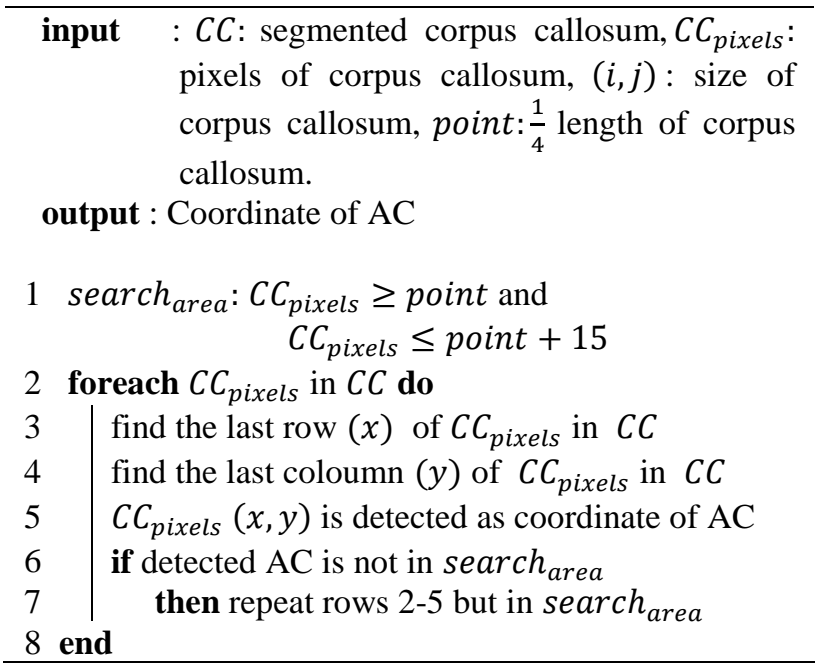

Table 4. Pseudocode for automatic detection of PC Algorithm 4: Automatic detection of PC

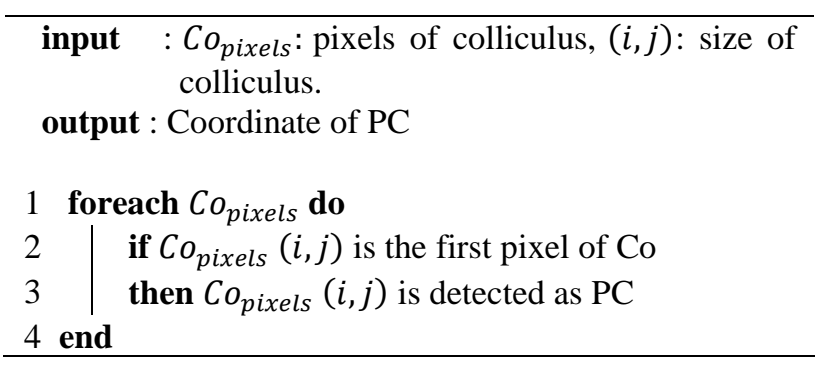


formed from point $(x-3, y-3)$ to $\operatorname{point}(x, y)$. The first pixel in the search region which value is greater than the second threshold will be considered as the new coordinate of detected PC. So that the coordinate position of $\mathrm{AC}$ and $\mathrm{PC}$ have been updated.

\section{Experiment and result}

In the experiments, 43 brain MRI data sets were used to validate the proposed method. All of the data are the MRI of adult patients with normal conditions without a tumour. The data sets consist of $15 \mathrm{~T} 1 \mathrm{~W}$ MRI and 28 T2W MRI with matrix sizes of $256 \times 256$ pixels and $512 \times 512$ pixels with various voxel sizes. The data used has been manually selected a midsagittal slice and the alignment process has been applied to certain images. The algorithm in this study does not require a training process so that the entire data will be used as data testing.

\subsection{Manual localization of AC and PC}

For evaluation of the algorithm, we manually located AC and PC on 43 mid-sagittal MRI data (15 T1W MRI and 28 T2W MRI). Manual detection of $\mathrm{AC}$ and PC were performed using a program that displayed three orthogonal slices. The localization of both landmarks generally started with a view of midsagittal MRI. AC generally found at the meeting point of corpus callosum and fornix. The arc of the commissure in the axial slice provided the signature of the AC [10]. While PC is located at the top of the superior colliculus, about $2 \mathrm{~mm}$ in diameter. In this study, all processes in manual detection of $\mathrm{AC}$ and $\mathrm{PC}$ are based on the doctor's direction. The example of manual localization of AC and PC based on midsagittal and axial slice is shown in Fig. 10.

\subsection{Results of experiment}

The algorithm was implemented in Matlab and 2 types of testing were performed to evaluate the accuracy of the algorithm. First, we compared manually segmented corpus callosum, fornix, and colliculus on 43 MRI data (15 T1W MRI and 28 T2W MRI) with the automatic segmentation by the algorithm using DICE similarity. Examples of the successful corpus callosum, fornix, and colliculus segmentation from mid-sagittal MRI and the comparison with the manual segmentation is shown in Fig. 11.

Second, we compared manual localization of AC and PC on 43 MRI data with the automatic detection by the algorithm. We used Euclidean distance in $\mathrm{mm}$ between the manual localization with the automatic detection of $\mathrm{AC}$ and $\mathrm{PC}$ to measure the error between

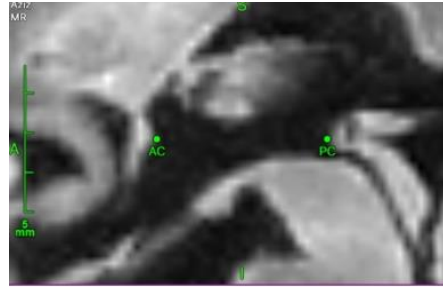

(a)

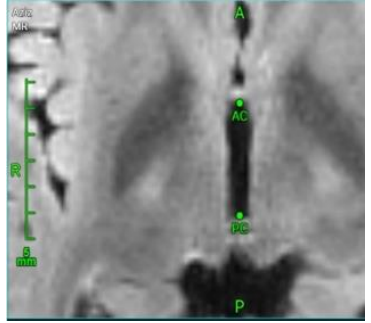

(b)
Figure. 10 Manual localization of AC and PC based on sagittal: (a) axial and (b) slice
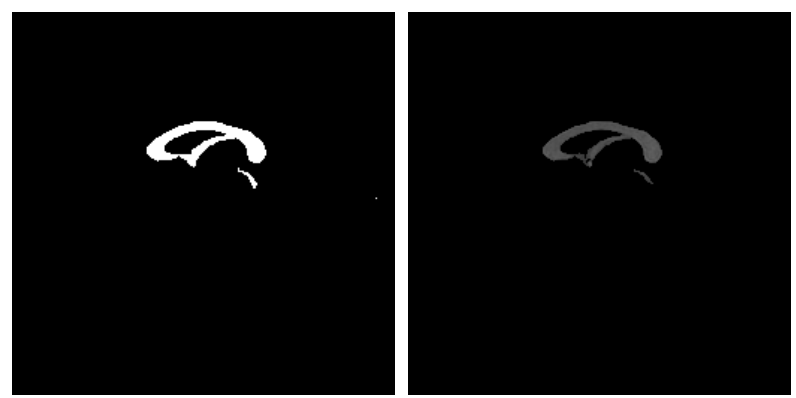

(a)
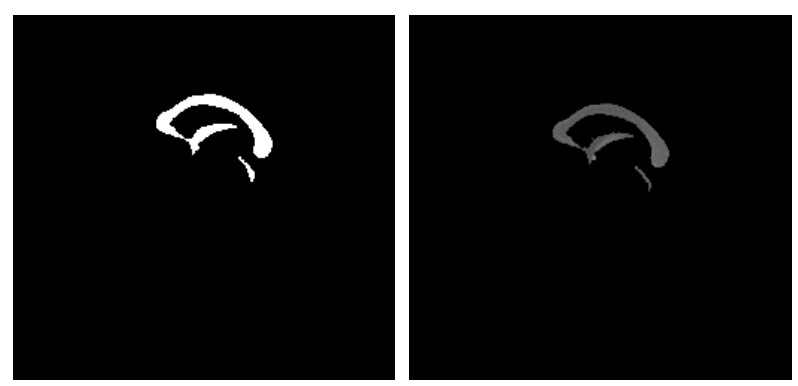

(b)
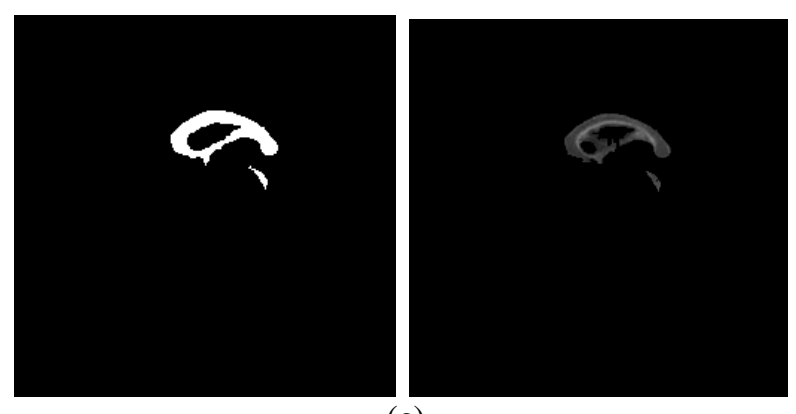

(c)

Figure. 11 Results of segmentation of corpus callosum, fornix, and colliculus. The left side is the ground truth while the right side is the automatic segmentation by the algorithm: (a) T1-W MRI with 512x512 pixel sizes, (b) T1-W MRI data with 256x256 pixel sizes, and (c) T2-W MRI data with 256x256 pixel sizes

the two. The result of the automatic detection of AC and PC and the comparison with the manual localization is shown in Fig. 12.

Based on testing, the accuracy of corpus callosum, fornix, and colliculus segmentation has been summarized in Table 5 and Table 6 . Table 5 shows the accuracy of the automatic segmentation of corpus 


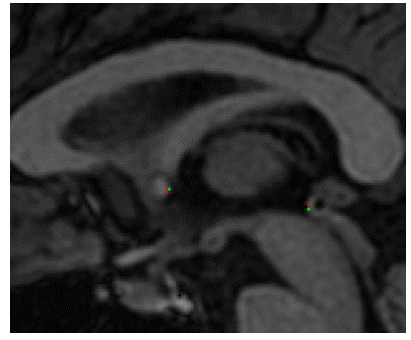

(a)

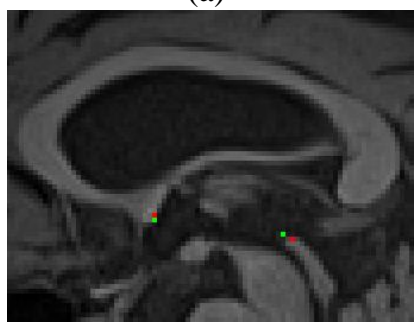

(c)

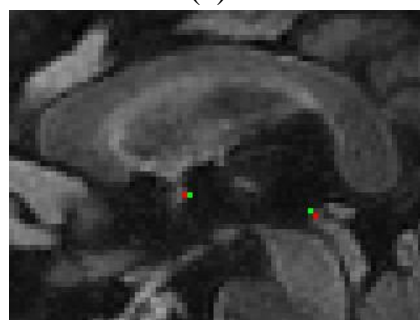

(e)

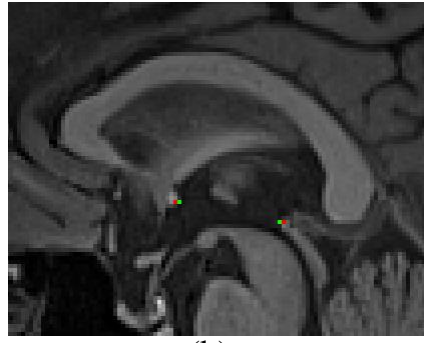

(b)

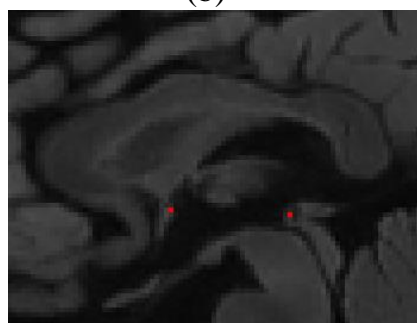

(d)

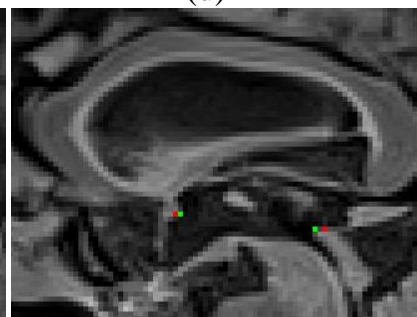

(f)
Figure. 12 Results of automatic detection of AC and PC. The green marker indicates the ground truth and the red marker indicates the automatic detection by the algorithm: (a) T1-W MRI with 512x512 pixel sizes, (b), and (c) T1-W MRI with 256x256 pixel sizes. (d), (e), and (f) T2-W MRI with 256x256 pixel sizes

Table 5. Accuracy of the automatic segmentation of corpus callosum and fornix

\begin{tabular}{|c|c|c|c|}
\hline \multirow{2}{*}{ Accuracy $(a)$} & \multicolumn{3}{|c|}{ Number of case } \\
\cline { 2 - 4 } & $\mathrm{T} 1-\mathrm{W}$ & $\mathrm{T} 2-\mathrm{W}$ & All data \\
\hline $50 \%<a \leq 70 \%$ & 0 & 3 & 3 \\
\hline $70 \%<a \leq 80 \%$ & 0 & 12 & 12 \\
\hline $80 \%<a \leq 90 \%$ & 0 & 10 & 10 \\
\hline$a>90 \%$ & 16 & 3 & 19 \\
\hline Average (\%) & $96.5 \%$ & $78.4 \%$ & $85 \%$ \\
\hline
\end{tabular}

Table 6. Accuracy of automatic segmentation of colliculus

\begin{tabular}{|c|c|c|c|}
\hline \multirow{2}{*}{ Accuracy $(a)$} & \multicolumn{3}{|c|}{ Number of case } \\
\cline { 2 - 4 } & T1-W & T2-W & All data \\
\hline $50 \%<a \leq 70 \%$ & 0 & 1 & 1 \\
\hline $70 \%<a \leq 80 \%$ & 3 & 4 & 7 \\
\hline $80 \%<a \leq 90 \%$ & 6 & 9 & 15 \\
\hline$a>90 \%$ & 7 & 14 & 21 \\
\hline Average $(\%)$ & $87.8 \%$ & $88.1 \%$ & $87.9 \%$ \\
\hline
\end{tabular}

Table 7. Statistics of errors between automatically and manually detected AC

\begin{tabular}{|c|c|c|c|}
\hline \multirow{2}{*}{ Error $(\varepsilon)$} & \multicolumn{3}{|c|}{ Number of case } \\
\cline { 2 - 4 } & T1-W & T2-W & All data \\
\hline \multicolumn{4}{|c|}{ ACtection } \\
\hline$\varepsilon<1 \mathrm{~mm}$ & 14 & 18 & 32 \\
\hline $1 \mathrm{~mm}<\varepsilon \leq 2 \mathrm{~mm}$ & 1 & 6 & 7 \\
\hline $2 \mathrm{~mm}<\varepsilon \leq 3 \mathrm{~mm}$ & 0 & 3 & 3 \\
\hline$\varepsilon>3 \mathrm{~mm}$ & 0 & 1 & 1 \\
\hline Average $(\mathrm{mm})$ & 0.77 & 1.16 & 1.02 \\
\hline \multicolumn{4}{|c|}{ PC detection } \\
\hline$\varepsilon<1 \mathrm{~mm}$ & 10 & 17 & 27 \\
\hline $1 \mathrm{~mm}<\varepsilon \leq 2 \mathrm{~mm}$ & 4 & 7 & 11 \\
\hline $2 \mathrm{~mm}<\varepsilon \leq 3 \mathrm{~mm}$ & 1 & 2 & 3 \\
\hline$\varepsilon>3 \mathrm{~mm}$ & 0 & 2 & 2 \\
\hline Average $(\mathrm{mm})$ & 0.71 & 1.25 & 1.06 \\
\hline
\end{tabular}

callosum and fornix with the respect to the ground truth, while Table 6 shows the accuracy of the automatic segmentation of colliculus with respect to the ground truth. The mean accuracy of the corpus callosum and fornix segmentation for all images was 85\%. $96.5 \%$ for T1-W and $78.4 \%$ for T2-W. While the mean accuracy of colliculus segmentation for all images was $87.9 \% .87 .8 \%$ for $\mathrm{T} 1-\mathrm{W}$ and $88.1 \%$ for T2-W.

The error statistics for the automatic detection of $\mathrm{AC}$ and $\mathrm{PC}$ with respect to the ground truth are shown in Table 7. The mean error of AC detection for all images was $1.02 \mathrm{~mm}, 0.77 \mathrm{~mm}$ for T1-W and 1.16 $\mathrm{mm}$ for T2-W. For PC detection, the mean error was $1.06 \mathrm{~mm}$ for all images, $0.71 \mathrm{~mm}$ for $\mathrm{T} 1-\mathrm{W}$ and 1.25 $\mathrm{mm}$ for $\mathrm{T} 2-\mathrm{W}$.

The results of the proposed method then compared with the previous researches [9-12] showed in Table 8. In this case, the test is not repeated using the same data because the whole methods are proposed a strategy, so it is quite complicated to implement and there is a possibility that the implementation is not in accordance with the implementation that has been done by previous researchers. The values listed in Table 8 are the results of the accuracy written on each paper. However, the data used in this study are sufficiently representative since it uses a more diverse type and matrix sizes of MRI. Further discussion regarding Table 8 will be explained in the next chapter.

\section{Discussion}

Quantitative evaluation of the algorithm to automatic segmentation of corpus callosum and fornix in Table 5 showed that the accuracy on T1-W 
Table 8. Comparison between the proposed method and the previous researches

\begin{tabular}{|c|c|c|c|c|}
\hline \multirow{2}{*}{ Method } & \multirow{2}{*}{$\begin{array}{l}\text { Type of } \\
\text { data }\end{array}$} & \multirow{2}{*}{$\begin{array}{c}\text { Matrix } \\
\text { sizes }\end{array}$} & \multicolumn{2}{|c|}{ Mean error } \\
\hline & & & $\mathrm{AC}$ & $\mathrm{PC}$ \\
\hline Segmentation Approach [9] & $\mathrm{T} 2-\mathrm{W}$ & $320 \times 248$ & $1.5 \mathrm{~mm}$ & $1.6 \mathrm{~mm}$ \\
\hline Model-Based Approach [10] & $\begin{array}{l}\text { T1-W } \\
\text { T2-W }\end{array}$ & $256 \times 256$ & $1.1 \mathrm{~mm}$ & $1.1 \mathrm{~mm}$ \\
\hline Random Forest Regression [11] & $\mathrm{T} 1-\mathrm{W}$ & $256 \times 256$ & $0.84 \mathrm{~mm}$ & $0.83 \mathrm{~mm}$ \\
\hline Deep Neural Network [12] & - & - & 0.85 pixel & 0.85 pixel \\
\hline Proposed method & $\begin{array}{l}\mathrm{T} 1-\mathrm{W} \\
\mathrm{T} 2-\mathrm{W}\end{array}$ & $\begin{array}{l}256 \times 256 \\
512 \times 512 \\
\end{array}$ & $1.02 \mathrm{~mm}$ & $1.04 \mathrm{~mm}$ \\
\hline
\end{tabular}

data was higher than those on $\mathrm{T} 2-\mathrm{W}$ data, with accuracy values were greater than $90 \%$ for all data sets. While in T2-W data, the majority of data sets have an accuracy value between $70-80 \%$, with the average accuracy value was $85 \%$. The fact that the accuracy of T2-W data sets is generally lower may be attributed to several factors: (1) In general, T1-W data has a better contrast than $\mathrm{T} 2-\mathrm{W}$, so the use of multilevel thresholding for automatic segmentation on $\mathrm{T} 2-\mathrm{W}$ tends to be more difficult. (2) In the majority of T2-W data, the contrast between objects with the background tends to be low. So that in some data, pixels in the hole in the middle of the corpus callosum are segmented (as shown in Fig. 10 (c)) which causes the accuracy to decrease.

Quantitative evaluation of the algorithm for automatic detection of AC and PC in Table 7 showed the mean error of $1.02 \mathrm{~mm}$ and $1.06 \mathrm{~mm}$ over all data sets, respectively. The mean error of both AC and PC is higher on T2-W data since the accuracy of the automatic segmentation of corpus callosum, fornix, and colliculus in $\mathrm{T} 2-\mathrm{W}$ is lower than $\mathrm{T} 1-\mathrm{W}$, which results of the segmentation affects to the determination of the coordinates of AC and PC. Error value of $\mathrm{AC}$ detection for all data sets is under $3 \mathrm{~mm}$, except 1 case with an error of $3.5 \mathrm{~mm}$. While in PC detection, 2 cases give an error value of $5.7 \mathrm{~mm}$ and $6.3 \mathrm{~mm}$. For these cases, we concluded that the algorithm failed for both types of data.

Previous research conducted by [9] reported that the mean errors of $20 \mathrm{~T} 2-\mathrm{W}$ MRI data sets were 1.5 $\mathrm{mm}$ for $\mathrm{AC}$ and $1.6 \mathrm{~mm}$ for $\mathrm{PC}$ with the voxel sizes of each data set was $0.72 \times 0.72 \times 1.50 \mathrm{~mm}^{3}$ and 320 $\mathrm{x} 248$ pixel sizes. The error value given is higher than the proposed method even the algorithm is only compatible on T2-W MRI data with the same matrix sizes, showed that it cannot handle the diversity of MRI. The model-based approach for automatic detection of AC and PC [10] gives the mean error of $1.1 \mathrm{~mm}$ for both landmarks on $126 \mathrm{MRI}$ data sets with
$256 \times 256$ pixel sizes. This method is compatible for T1-W and T2-W MRI data. But since it was a modelbased approach, it requires a large enough data sets with diverse variations to obtain a maximum result and requires a time-consuming training process. From the comparison in Table 8 , the algorithm conducted by [11] gives the best results in which the mean error is lower than the proposed method, 0.84 $\mathrm{mm}$ for $\mathrm{AC}$ and $0.83 \mathrm{~mm}$ for PC. Although it gives the best results, the paper stated that all of the data sets are MRI data with similar poses, low orientation differences, and low position differences. In addition, the training process that requires diversity of MRI is also needed [11]. Based on test conducted by [12] using Deep Neural Network, the mean error value for both landmarks on 143 mid-sagittal MRI is $0.85 \mathrm{~mm}$ without any specification regarding the error value for AC and the error value for PC. Also, the type of MRI used is not mentioned, whether using T1-W or $\mathrm{T} 2-\mathrm{W}$ and the specification of its matrix size. Thus, the reliability of the algorithm on the diversity of MRI cannot be determined. Although the mean error value is quite good, the use of neural networks has several drawbacks such as a hardware dependence, requires a large enough dataset to produce a good training model, as well as the need for a timeconsuming training process.

The mean error of $\mathrm{AC}$ and $\mathrm{PC}$ produced by the proposed method is higher than $[11,12]$, but better than [9] and [10] with the mean error of AC and PC were $1.02 \mathrm{~mm}$ and $1.06 \mathrm{~mm}$, respectively. Proposed method using a multilevel thresholding and the morphological relationship approach has succeeded in detecting $\mathrm{AC}$ and $\mathrm{PC}$ since according to expert, the acceptable stereotactic targeting in neurosurgery is under $1.5 \mathrm{~mm}$. In addition, it performs an automatic detection of AC and PC with a simple algorithm, does not require a large of diverse data sets for the training process, without training process that takes up time, and reliable on the diversity of MRI since it is 
compatible for $\mathrm{T} 1-\mathrm{W}$ and $\mathrm{T} 2-\mathrm{W}$ with various matrix sizes of 256 x 256 and 512 x 512 pixels which cannot be handled by previous researches.

\section{Conclusion}

We presented a new strategy for the automatic detection of AC and PC on mid-sagittal MRI using a multilevel thresholding and morphological relationship approach. Automatic segmentation of corpus callosum, fornix, and colliculus are first performed to determine the location of $\mathrm{AC}$ and $\mathrm{PC}$. The algorithm was implemented in Matlab and used two types of testing to evaluate the accuracy of the algorithm. First, we compared the manually segmented of corpus callosum, fornix, and colliculus on 43 MRI data using DICE similarity. Then, we compared the manual localization of $\mathrm{AC}$ and $\mathrm{PC}$ with automatic detection by the algorithm using Euclidean distance. Test results presented that the mean error of corpus callosum and fornix segmentation was $85 \%$, while the mean error of colliculus was $87.9 \%$. The algorithm was also successfully detecting AC and PC with the mean error were $1.02 \mathrm{~mm}$ and $1.06 \mathrm{~mm}$, respectively. According to expert, the acceptable stereotactic targeting in neurosurgery is under 1.5 $\mathrm{mm}$ which is shows that the proposed method was successful. In addition, it performs an automatic detection of $\mathrm{AC}$ and $\mathrm{PC}$ with a simple algorithm, does not require a large of diverse data sets for the training process, without training process that takes up time, and reliable on the diversity of MRI since it is compatible for T1-W and T2-W with various matrix sizes of $256 \times 256$ and $512 \times 512$ pixels which cannot be handled by previous researches.

\section{Conflicts of Interest}

The authors declare no conflict of interest.

\section{Author Contributions}

The first author, Khairiyyah Nur Aisyah contributed in the formulation of methods, the implementation of algorithm, conduct of experiments, and the formation of the paper. Chastine Fathichah and Riyanarto Sarno as supervisors propose ideas of problems, supervised the formulation methods for solving problems, and checked the paper.

\section{Acknowledgments}

The authors would like to sincerely thank Institut Teknologi Sepuluh Nopember, the Directorate of Higher Education, Indonesian Ministry of Education and Culture, and LPDP through RISPRO Invitation Program for funding the research.

\section{References}

[1] S. H. Choi, J. G. Chi, Y. B. Kim, and Z. H. Cho, "Anterior Commissure - Posterior Commissure Revisited", Korean Journal of Radiology, Vol. 14, No. 4, pp. 653-661, 2013.

[2] Y. Duan, X. Fan, H. Cheng, and H. Kang, "G Gradient Regression for Brain Landmark Localization on Magnetic Resonance Imaging", In: Proc. of 2018 25th IEEE International Conf. on Image Processing (ICIP), pp. 4013-4017, 2018.

[3] J.-J. Lemaire, A. D. Salles, G. Coll, Y. el Ouadih, R. Chaix, J. Coste, F. Durif, N. Makris, and R. Kikinis, "MRI Atlas of the Human Deep Brain", Frontiers in Neurology, Vol. 10, 2019.

[4] P. L. Bazin, J. L. Cuzzocreo, M. Yassa, W. Gandler, M. Mcauliffe, S. S. Bassett, and D. L. Pham, "Volumetric Neuroimage Analysis Extensions for the MIPAV Software Package", Journal of Neuroscience Methods, Vol. 165, No. 1, pp. 111-121, 2007.

[5] P. A. Starr, A. J. Martin, J. L. Ostrem, P. Talke, N. Levesque, and P. S. Larson, "Subthalamic Nucleus Deep Brain Stimulator Placement using High-Field Interventional Magnetic Resonance Imaging and a Skull-Mounted Aiming Device: Technique and Application Accuracy", Journal of Neurosurgery, Vol. 112, No. 3, pp. 479-490, 2010.

[6] N. Desai, A. S. Rao, P. Palaniswami, D. Thyagarajan, and M. Palaniswami, "Arytenoid Cartilage Feature Point Detection using Laryngeal 3D CT Images in Parkinson's disease", In: Proc. of the Annual International Conf. of the IEEE Engineering in Medicine and Biology Society, pp. 1820-1823, 2017.

[7] S. Pallavaram, H. Yu, J. Spooner, P. D'haese, T. Koyama, B. Bodenheimer, P. Konrad, and B. M. Dawant, "Automated Selection of Anterior and Posterior Commissures based on a Deformable Atlas and Its Evaluation Based on Manual Selections by Neurosurgeons", SPIE Medical Imaging: Visualization and Image-Guided Procedures, Vol. 6509, pp. 65091C, 2007.

[8] K. N. Bhanu Prakash, Q. Hu, A. Aziz, and W. L. Nowinski, "Rapid and Automatic Localization of The Anterior and Posterior Commissure Point Landmarks in MR Volumetric Neuroimages", Academic Radiology, Vol. 13, No. 1, pp. 36-54, 2006.

[9] G. Zhang, H. Xu, S. Wang, and W. Gao, "Automatic Localization of $\mathrm{AC}$ and $\mathrm{PC}$ Landmarks in T2-weighted MR Volumetric Neuroimages", In: Proc. of 2010 IEEE 
International Conf. on Information and Automation (ICIA) pp. 1830-1834, 2010.

[10] B. A. Ardekani and A. H. Bachman, "ModelBased Automatic Detection of The Anterior and Posterior Commissures on MRI Scans", NeuroImage, Vol. 46, No. 3, pp. 677-682, 2009.

[11] Y. Liu and B. M. Dawant, "Automatic Localization of the Anterior Commissure, Posterior Commissure, and Midsagittal Plane in MRI Scans using Regression Forests”, IEEE Journal of Biomedical and Health Informatics, Vol. 19, No. 4, pp. 1362-1374, 2015.

[12] X. Yang, W. T. Tang, G. Tjio, S. Y. Yeo, and Y. $\mathrm{Su}$, "Automatic Detection of Anatomical Landmarks in Brain MR Scanning using MultiTask Deep Neural Networks", Neurocomputing, Vol. 396, pp. 514-521, 2020.

[13] Vijayalaxmi, M. Fatahi, and O. Speck, "Magnetic Resonance Imaging (MRI): A Review of Genetic Damage Investigations", Mutation Research - Reviews in Mutation Research, Vol. 764, pp. 51-63, 2015.

[14] Z. Kikinis, J. Fitzsimmons, C. Dunn, M. Vu, N. Makris, S. Bouix, J. M. Goldstein, R. I. MGately, T. Petryshen, E. C. del Re, J. Wojcik, L. J. Seidman, and M. Kubicki, "Anterior Commissural White Matter Fiber Abnormalities in First-Episode Psychosis: A Tractography Study", Schizophrenia Research, Vol. 162, No. 1-3, pp. 29-34, 2015.

[15] N. G. Ozdemir, "The Anatomy of the Posterior Commissure", Turkish Neurosurgery, Vol. 25, No. 6, pp. 837-843, 2015.

[16] A. Fitsiori, D. Nguyen, A. Karentzos, J. Delavelle, and M. I. Vargas, "The Corpus Callosum: White Matter or Terra Incognita", British Journal of Radiology, Vol. 84, No. 997, pp. 5-18, 2011.

[17] A. Chandra, S. Verma, A. S. Raghuvanshi, N. D. Londhe, N. K. Bodhey, and K. Subham, "Corpus Callosum Segmentation from Brain MRI and its Possible Application in Detection of Diseases", In: Proc. of 2019 3rd IEEE International Conf. on Electrical, Computer and Communication Technologies (ICECCT), 2019.

[18] F. D. Raslau, J. C. Augustinack, A. P. Klein, J. L. Ulmer, V. P. Mathews, and L. P. Mark, "Memory Part 3: The Role of The Fornix and Clinical Cases", American Journal of Neuroradiology, Vol. 36, No. 9, pp. 1604-1608, 2015.

[19] V. Douet and L. Chang, "Fornix as an Imaging Marker for Episodic Memory Deficits in Healthy Aging and in Various Neurological
Disorders", Frontiers in Aging Neuroscience, Vol. 7, pp. 1-19, 2015.

[20] M. A. Basso and P. J. May, "Circuits for Action and Cognition: A View from the Superior Colliculus", Annual Review of Vision Science, Vol. 3, No. 1, pp. 197-226, 2017.

[21] M. S. Malmierca and E. D. Young, "Inferior Colliculus Microcircuits", Frontiers in Neural Circuits, Vol. 8, pp. 3389, 2014.

[22] U. Salamah, R. Sarno, A. Z. Arifin, Sarimuddin, A. S. Nugroho, I. E. Rozi, and P. B. S. Asih, "Segmentation of Malaria Parasite Candidate from Thickblood Smear Microscopic Images using Watershed and Adaptive Thresholding", Journal of Telecommunication, Electronic and Computer Engineering, Vol. 10, No. 2-4, pp. 113-117, 2018.

[23] U. Salamah, R. Sarno, A. Z. Arifin, A. S. Nugroho, I. E. Rozi, and P. B. S. Asih, "A Robust Segmentation for Malaria Parasite Detection of Thick Blood Smear Microscopic Images", International Journal on Advanced Science, Engineering and Information Technology, Vol. 9, No. 4, pp. 1450-1459, 2019.

[24] U. Salamah, R. Sarno, A. Z. Arifin, A. S. Nugroho, I. E. Rozi, and P. B. S. Asih, "Incorporating Index of Fuzziness and Adaptive Thresholding for Image Segmentation", International Journal of Electrical and Computer Engineering, Vol. 8, No. 4, pp. 24062418, 2018.

[25] P. Smith, D. B. Reid, C. Environment, L. Palo, P. Alto, and P. L. Smith, "A Threshold Selection Method from Gray-Level Histograms", Vol. 20, No. 1, pp. 62-66, 1979. 